\title{
Abordagem do lúpus eritematoso sistêmico em gestantes: revisão narrativa
}

\author{
Approach of systemic lupus erythematosus in pregnant women: narrative review
}

Abordaje del lupus eritematoso sistémico en embarazadas: revisión narrativa

Amanda Brandão Lopes ${ }^{1 *}$, Izadora Marina de Oliveira Sabino ${ }^{1}$, João Pedro Soares Rodrigues ${ }^{1}$, Ayla Silva Sygilião Ribeiro ${ }^{1}$, Arthur Souto Maior Filizzola ${ }^{1}$, Thiago Rodrigues Ferreira ${ }^{1}$, Renata Silva de Abreu$^{1}$, Fernanda Dias Pinheiro ${ }^{2}$, Karolyne Mariano Abrantes ${ }^{3}$, Noelly Silva Borburema ${ }^{1}$.

\section{RESUMO}

Objetivo: Revisar e fornecer uma ampla abordagem sobre o lúpus eritematoso sistêmico durante a gestação e disponibilizar informações atualizadas para que possam ser alcançados melhores desfechos em gestações de alto risco, alcançando um melhor prognóstico materno-fetal. Revisão Bibliográfica: O Lúpus Eritematoso Sistêmico (LES) é a doença autoimune mais comum na gravidez. Sua clínica é bastante variável e, como os sinais e sintomas são muitas vezes inespecíficos, ele pode mimetizar outras doenças e dificultar diagnóstico. É uma doença que pode afetar diversos órgãos em diferentes graus. Como o LES durante a gestação aumenta os riscos maternos e fetais, é importante solicitar exames laboratoriais para melhor controle da gestante. O monitoramento da gravidez deve ser multidisciplinar e inclui avaliação clínica, laboratorial e ultrassonográfica regular. Antes de engravidar, idealmente, a gestação deverá ser planejada. Considerações finais: O lúpus pode trazer malefícios à gestação e ao bebê, assim como a gestação pode exacerbar o lúpus, portanto, recomenda-se à gestante acompanhamento médico rigoroso pré e pós concepção e principalmente durante a gravidez.

Palavras-chave: Lúpus eritematoso sistêmico, Gravidez, Doença autoimune.

\begin{abstract}
Objective: Review and provide a comprehensive approach to systemic lupus erythematosus during pregnancy and provide updated information so that better outcomes can be achieved in high-risk pregnancies, achieving a better maternal-fetal prognosis. Literature Review: Systemic Lupus Erythematosus (SLE) is the most common autoimmune disease in pregnancy. Its clinical features are quite variable and, as the signs and symptoms are often nonspecific, it can mimic other diseases and make diagnosis more difficult. It is a disease that can affect different organs to different degrees. As SLE during pregnancy increases maternal and fetal risks, it is important to request laboratory tests for better control of the pregnant woman. Pregnancy monitoring should be multidisciplinary and include regular clinical, laboratory and ultrasound evaluation. Before getting pregnant, ideally, the pregnancy should be planned. Final considerations: Lupus can harm pregnancy and the baby, as well as pregnancy can exacerbate lupus, therefore, it is recommended to pregnant women rigorous medical monitoring before and after conception and especially during pregnancy.
\end{abstract}

Key words: Systemic lupus erythematosus, Pregnancy, Autoimmune disease.

\section{RESUMEN}

Objetivo: Revisar y brindar un abordaje integral del lupus eritematoso sistémico durante el embarazo y brindar información actualizada para que se puedan lograr mejores resultados en embarazos de alto riesgo, logrando un mejor pronóstico materno-fetal. Revisión de la literatura: El Lupus Eritematoso Sistémico (LES) es la enfermedad autoinmune más común en el embarazo. Sus características clínicas son bastante variables y, como los signos y síntomas a menudo son inespecíficos, puede simular otras enfermedades y dificultar el

\footnotetext{
${ }^{1}$ Faculdade de Minas de Belo Horizonte (FAMINAS-BH), Belo Horizonte - MG.

*E-mail: amandabrandaolopes@gmail.com

${ }^{2}$ Centro Universitário Facisa (Unifacisa), Campina Grande - PB.

${ }^{3}$ Universidade de Itaúna (UIT), Itaúna - MG.
} 
diagnóstico. Es una enfermedad que puede afectar diferentes órganos en diferentes grados. Dado que el LES durante el embarazo aumenta los riesgos maternos y fetales, es importante solicitar pruebas de laboratorio para un mejor control de la gestante. El seguimiento del emba,razo debe ser multidisciplinario e incluir evaluaciones clínicas, de laboratorio y ecográficas periódicas. Antes de quedar embarazada, lo ideal es planificar el embarazo. Consideraciones finales: El lupus puede dañar el embarazo y al bebé, así como el embarazo puede exacerbar el lupus, por ello, se recomienda a las mujeres embarazadas un riguroso seguimiento médico antes y después de la concepción y especialmente durante el embarazo.

Palabras clave: Lupus eritematoso sistémico, Embarazo, Enfermedad autoinmune

\section{INTRODUÇÃO}

O Lúpus Eritematoso Sistêmico (LES) afeta predominantemente mulheres em idade reprodutiva. Há uma interação imunológica complexa durante a gravidez em pacientes com lúpus. A gravidez tem impacto direto sobre a doença, onde um aumento na taxa de crises é observado. Gestantes com LES apresentam risco aumentado para parto prematuro, restrição de crescimento intrauterino (CIUR), perda fetal e pré-eclâmpsia, além dessa doença estar associada à maior morbimortalidade materna (MAYNARD S, et al., 2019).

As complicações potenciais incluem crises de lúpus, complicações obstétricas e síndrome do lúpus neonatal. Alguns fatores estão diretamente relacionados ao aumento do risco de eventos adversos na gestação, como por exemplo a síndrome antifosfolípide e a presença de relações com anticorpos antifosfolípides. Os anticorpos anti-SSA e/ou anti-SSB colocam os fetos em risco de lúpus neonatal (IMBERT GG, et al., 2016).

As reações adversas que o lúpus gestacional comumente provoca, podem ser minimizados pelo uso de alguns medicamentos aprovados durante a gravidez, como os corticóides, a hidroxicloroquina e a azatioprina. Para aquelas complicações que levam a doenças hipertensivas, à gestante será direcionada a uma terapia apropriada e, em casos de pacientes com síndromes antifosfolípides, o uso de anticoagulantes pode se fazer necessário (DALAL DS, et al., 2019).

Melhorar o resultado dessas gestações depende de um planejamento sistemático da gravidez em uma consulta de aconselhamento pré-concepção, com definição do envolvimento clínico e sorológico do lúpus juntamente com uma abordagem multidisciplinar. A ausência de atividade lúpica, o uso de medicação apropriada durante a gestação com base no histórico médico e nos fatores de risco juntamente com o monitoramento regular constituem as melhores ferramentas para alcançar um resultado favorável (BĂLĂNESCU A, et al., 2017).

O objetivo desta revisão é fornecer uma ampla abordagem sobre o lúpus eritematoso sistêmico durante a gestação a fim disponibilizar informações atualizadas para que possam ser alcançados melhores desfechos em gestações de alto risco, alcançando um melhor prognóstico materno-fetal.

\section{REVISÃO BIBLIOGRÁFICA}

\section{Características gerais do lúpus eritematoso sistêmico}

O lúpus eritematoso sistêmico é a doença autoimune crônica multissistêmica mais comum na gravidez. Sua clínica é bastante variável, incluindo erupção cutânea, artrite, anemia, trombocitopenia, serosite, nefrite, convulsões e psicose (KNIGHT CL e PIERCY CN, 2017). Pode estar associada a alterações imunológicas como complementos baixos, DNA de fita dupla positivo, antígeno nuclear extraível e presença de anticorpos antifosfolipídes (DALAL DS, et al., 2019).

O LES possui sinais e sintomas pouco específicos, podendo mimetizar variadas doenças sistémicas, o que acaba por dificultar o seu diagnóstico. Épocas de remissão seguidas por períodos de maior atividade são características do padrão de evolução da doença (HOCHBERG MC, 2017).

Gestantes portadoras de LES apresentam cerca de 2 a 4 vezes mais complicações obstétricas do que gestantes sem essa patologia, o que aumenta aproximadamente 20 vezes o risco de mortalidade materna 
(HOCHBERG MC, 2017). Como o LES durante a gestação aumenta os riscos maternos e fetais, é importante solicitar exames laboratoriais como níveis de complemento, anticorpos anti-DNA de cadeia dupla (dsDNA), anticorpos anticardiolipina, anticoagulante lúpico, anti-SSA e anti-SSB (anti-Ro e anti-La) e estudos de urina de 24 horas para haver melhor controle da doença na gestante (OSTENSE M, et al., 2016).

\section{Efeitos do lúpus eritematoso sistêmico na gestação}

A complicação mais comum decorrente do LES na gestação é o parto prematuro que pode ocorrer em aproximadamente um terço das pacientes. Dentre os fatores de risco para prematuros estão: o aumento da atividade da doença (em termos clínicos e sorológicos), aumento do uso de prednisona (o que pode causar ruptura prematura de membranas) e hipertensão secundária (MAYNARD S, et al., 2019).

Pacientes com lúpus têm uma alta taxa de complicações durante a gravidez, incluindo pré-eclâmpsia, que afeta $16 \%$ a $30 \%$ das mulheres com LES em comparação com $5 \%$ a $7 \%$ das mulheres saudáveis. Fatores que favorecem o desenvolvimento dessa condição são: o próprio lúpus, nefrite lúpica específica, alteração dos marcadores de doença, presença de anticorpos antifosfolipídes, trombocitopenia e redução dos níveis de complemento, além de outros fatores predisponentes, como idade materna avançada, história de doença hipertensiva na gravidez anterior, hipertensão arterial sistêmica, diabetes e obesidade preexistentes. A préeclâmpsia representa um desafio clínico único, dado a semelhança entre pré-eclâmpsia e nefrite lúpica, ambas caracterizadas por deterioração da função renal, aumento da proteinúria, hipertensão e plaquetopenia (BETZ RF e SPWCKER C, 2017).

Anticorpos antifosfolipídes ocorrem em cerca de um quarto a metade das pacientes com lúpus. Notavelmente, esses anticorpos podem ocorrer sem lúpus coexistente (síndrome antifosfolipídica primária) e representam o mesmo risco para a gestação. A presença de anticorpos antifosfolipídios sem a síndrome aumenta o risco de resultados adversos da gravidez como retardo de crescimento intrauterino, partos prematuros e perdas gestacionais. Acredita-se que a fisiopatologia esteja relacionada à trombose na vasculatura uterina, bem como ligação de anticorpos a trofoblastos, células endoteliais e neuronais (ANDREOLI L, et al., 2017).

\section{Efeitos da gestação no lúpus eritematoso sistêmico}

Na gravidez normal, as alterações imunológicas permitem a tolerância materna do feto. Há um aumento de células T reguladoras CD41/CD251 (Tregs), bem como uma mudança de Th1 (pró-inflamatório) para Th2 (anti-inflamatório). Essa mudança pode aumentar a atividade de doenças mediadas por Th2, incluindo lúpus. O LES também pode levar a uma alteração funcional ou quantitativa na população Tregs (MAYNARD S, et al., 2019).

Mudanças hormonais durante a gestação também podem afetar a atividade lúpica. Em um modelo de estudo sobre LES, foi comprovado que o estrogênio acelera a glomerulonefrite, a linfoproliferação e as taxas de mortalidade na doença (JEON C, et al., 2017).

O início da gestação constitui um fator de risco para a progressão dessa patologia. O risco de doença ativa é praticamente idêntico, independentemente do trimestre e continua o mesmo no período pós-parto. Episódios são geralmente de gravidade leve a moderada, com sinais articulares sendo menos comuns, enquanto sinais renais e hematológicos são mais comuns e mais graves (IMBERT GG, et al., 2016).

Pacientes com história prévia de nefrite lúpica possuem maior risco de novos episódios, especialmente no caso de sequelas persistentes envolvendo proteinúria ou insuficiência renal. Além disso, o aumento do fluxo de sangue natural da gestação pode acelerar o processo de degradação da função renal (WU J, et al., 2018).

\section{Lúpus neonatal}

O lúpus neonatal é uma condição temporária que dura aproximadamente de 6 a 8 meses após o nascimento. Patologicamente deve-se adquirir passivamente a autoimunidade de autoanticorpos maternos que atravessam a placenta e, portanto, duram enquanto os autoanticorpos maternos permanecem na circulação fetal (MAYNARD S, et al., 2019). 
Cerca de 30 a 40\% das mulheres com LES são positivas para autoanticorpos anti-Ro/SSA e 10 a $15 \%$ são positivas para autoanticorpos anti-La/SSB, colocando suas gestações em risco para lúpus neonatal. Esta síndrome pode causar de forma transitória e benigna manifestações cutâneas, hematológicas e hepáticas em 10 a 30\% dos recém-nascidos (MARDER W, 2019).

O aparecimento de manifestações cutâneas pode ocorrer logo depois do nascimento, mas na grande maioria dos casos, aparecem após as primeiras semanas de vida. Um dos exemplos dessas manifestações é o surgimento de placas eritematosas anulares ou policísticas com ou sem escamas finas que podem surgir no pescoço, no couro cabeludo ou na face, normalmente com distribuição periorbital, que podem ser chamadas de "olho de guaxinim" ou "olho de coruja". Apesar de não ser tão comum, no tronco ou nas extremidades também podem surgir placas de aspecto bastante semelhante. As lesões podem ser descamativas, urticariformes, crostosas ou ulcerativas. As lesões bolhosas, por sua vez, podem ser vistas na maior parte das vezes na planta dos pés (HON KL e LEUNG AKC, 2012).

Em casos de acometimento hepatobiliar, os sinais clínicos podem incluir hepatite colestática, insuficiência hepática, hepatomegalia e esplenomegalia, sendo esta última menos comum e, ambas transitórias na grande maioria dos casos. Essas situações clínicas podem levar em algumas semanas ou meses após o nascimento a elevação das enzimas hepáticas (como TGO-AST e TGP-ALT) e a um quadro de hiperbilirrubinemia conjugada (HON KL e LEUNG AKC, 2012).

Nas primeiras semanas de vida da criança podem ocorrer alguns distúrbios hematológicos devido ao LES, como anemia hemolítica, neutropenia e trombocitopenia, sendo o principal sinal clínico desta o aparecimento de petéquias na pele. As manifestações clínicas decorrentes destes distúrbios comumente iniciam na segunda semana de vida e duram até o final do segundo mês. Normalmente esses casos são assintomáticos. A neutropenia é causada devido à ligação de autoanticorpos, como anticorpo anti-Ro, com os neutrófilos. A linfopenia, por sua vez não é um distúrbio hematológico característico do lúpus, apesar de ser um achado relativamente comum em indivíduos com a doença (YOKOGAWA N, et al., 2017).

A complicação mais temida é o lúpus neonatal cardíaco, do qual o bloqueio cardíaco congênito é a manifestação mais comum, encontrada em cerca de $2 \%$ das gestações SSA/SSB positivas. Se uma mulher com SSA/SSB teve um bebê com lesão cutânea ou lúpus neonatal cardíaco, o risco do próximo apresentar um bloqueio cardíaco aumenta para 13-18\% nas futuras gestações (YOKOGAWA N, et al., 2017).

Aproximadamente $20 \%$ das crianças afetadas por bloqueio completo morrerá no útero ou no primeiro ano de vida e até $70 \%$ exigirá um marcapasso. Envolvimento além do sistema de condução também pode ser observado em doenças cardíacas do lúpus neonatal, incluindo fibroelastose endocárdica e cardiomiopatia dilatada, conferindo um pior prognóstico à doença (MARDER W, 2019).

Outras comorbidades que possuem chances de estarem associadas ao LES e podem ser citadas como exemplo de possíveis complicações são: hidrocefalia, macrocefalia, pneumonite, meningite asséptica e mielopatia, sendo essas duas últimas pouco relatadas na literatura. As manifestações clínicas mais comuns na pneumonite são taquipneia e taquicardia (HON KL e LEUNG AKC, 2012).

O lúpus eritematoso neonatal pode mimetizar diversas comorbidades, o que dificulta por muitas vezes o seu diagnóstico. Esse diagnóstico normalmente é feito tomando como base as características clínicas apresentadas pela criança juntamente com a demonstração de anticorpos associados no soro do bebê afetado ou no da mãe. Como diagnóstico diferencial podem ser citados: dermatite atópica, dermatite seborreica, acne neonatal, psoríase, tinea corporis, granuloma anular, histiocitose de células de Langerhans, eritema multiforme, rubéola congênita, sífilis congênita, síndrome de Rothmund-Thomso e síndrome de Bloom (HON KL e LEUNG AKC, 2012).

\section{Manejo medicamentoso da gestante com lúpus eritematoso sistêmico}

Em gestantes o uso de medicamentos deve ser baseado em dados de diversos estudos retrospectivos, relatos de casos isolados e estudos com animais. Normalmente as instruções de uso são tratadas com bastante cautela pelos fabricantes e órgãos administrativos de medicamentos, e geralmente são baseadas 
em uma série de pesquisas para que sejam evitadas complicações maternas e/ou fetais (KNIGHT CL e PIERCY CN, 2017)

Nas gestantes com lúpus os medicamentos que são considerados mais seguros em todos os trimestres da gestação e também durante a amamentação para tratar a exacerbação da doença ou manter a sua remissão são: hidroxicloroquina (HCQ), ciclosporina, azatioprina e tacrolimus. No manejo do LES deve ser dada a preferência para medicamentos imunossupressores poupadores de esteróides, como por exemplo a azatioprina. Idealmente devem ser sem esteróides ou possuírem uma dose de manutenção efetiva menor, com valor inferior a 7,5 mg de prednisolona ao dia. Corticosteroides, paracetamol e aspirina são fármacos seguros no controle dos sintomas ativos da doença. Existem alguns medicamentos que devem ser evitados ou interrompidos durante a gravidez e lactação por possuírem efeitos teratogênicos comprovados, são eles: metotrexato, ciclofosfamida e micofenolato de mofetil (KNIGHT CL e PIERCY CN, 2017).

As crises leves de LES durante a gestação podem ser tratadas com uso de corticoesteróides de baixa dosagem ou hidroxicloroquina. Além disso, se a gestante estiver no primeiro ou segundo trimestre de gestação também é possível o uso de anti-inflamatórios não esteroidais. Já em casos moderados ou graves da doença, o uso de metilprednisolona intravenosa pulsada, esteróides orais em altas doses ou imunoglobulina intravenosa podem ser as únicas opções terapêuticas disponíveis. Esta conduta de tratamento deve ser seguida de uma redução acelerada para baixas doses de esteróides orais de manutenção combinados com imunossupressores seguros (IMBERT GG, et al., 2016).

Nos casos graves da doença deve ser sempre realizada uma avaliação de risco e benefício para tomar condutas quanto a priorização da saúde materna diante das preocupações fetais. Em situações de alto risco para saúde da mãe, pode ser necessário a utilização de medicamentos mais agressivos, como ciclofosfamida ou micofenolato de mofetil, além de ser levada em consideração a possibilidade de interrupção da gestação em algumas situações. Em casos de nefrite lúpica durante a gestação o uso de esteroide pulsado deve ser avaliado e acompanhado por uma combinação com HCQ, prednisolona, azatioprina ou tacrolimo (IMBERT GG, et al., 2016).

Em casos de hipertensão ou pré-eclâmpsia pré-existentes ou induzidas pela gravidez, labetalol, metildopa e nifedipina são exemplos de anti-hipertensivos que podem ser considerados como tratamento seguro e de primeira linha. Eles podem ser utilizados de forma combinada ou isolada e em qualquer estágio da concepção, gestação ou lactação. A hidralazina e a doxazosina compõe os tratamentos de segunda linha durante a gravidez. Devido a um aumento no risco de desenvolvimento de anomalias congênitas os bloqueadores do receptor da angiotensina II e os inibidores da enzima de conversão da angiotensina (IECA) são exemplos de medicações que devem ser interrompidos até mesmo antes da concepção (KNIGHT CL e PIERCY CN, 2017)

Em alguns casos, o uso de IECA pode ser mantido até o início da gravidez, isto é, até um resultado de beta HCG positivo e, em seguida, alterado para outro anti-hipertensivo de primeira escolha. Isso acontece com algumas mulheres com doença renal em planejamento gestacional com orientação e supervisão de um especialista em que meses sem os efeitos protetores renais do IECA podem ser, à longo prazo, prejudiciais para à saúde materna. Essa classe medicamentosa pode ser utilizada de forma segura no pós-parto e também durante a lactação (DALAL DS, et al., 2019).

\section{Monitoramento da gestante com lúpus eritematoso sistêmico}

O monitoramento da gestante com lúpus deve ser multidisciplinar e inclui avaliação clínica, laboratorial e ultrassonográfica regular. Essa avaliação geralmente é realizada mensalmente e em intervalos mais curtos no final da gravidez, no entanto, é importante ressaltar que deve ser adaptado à história obstétrica da paciente e à progressão da gestação em questão (IMBERT GG, et al., 2016).

A triagem de rotina deve ser realizada por exame clínico e exames laboratoriais para sinais evocativos de episódios de exacerbação do lúpus, pré-eclâmpsia ou síndrome HELLP. O monitoramento de ultrassom consiste no ultrassom padrão para cada trimestre da gravidez. Dentro do bloco de exames, o ultrassom doppler do útero deve ser realizado, seguido de monitoramento de biometria fetal e nível de líquido amniótico. 
Ecocardiografia fetal atualmente é proposto para todas as mulheres com anticorpos anti-SSA ou anti-SSB positivos (IMBERT GG, et al., 2016).

\section{Planejamento da gestação da mulher com lúpus eritematoso sistêmico}

Houve declínio significativo nas taxas de perdas gestacionais em pacientes com lúpus em tratamento e acompanhamento adequado com mais de $80-90 \%$ das gestações resultando em nascidos vivos, o que representa uma grande melhora ao longo do tempo (DALAL DS, et al., 2019). Antes de engravidar, as mulheres com LES devem discutir seu desejo com a equipe médica precocemente e, idealmente, a gestação deverá ser planejada. Devem ser corretamente aconselhadas a evitar a gravidez se tiverem comorbidades de alto risco associadas ao lúpus (PASTORE DEA, et al., 2018).

Dentre as comorbidades de alto risco estão: hipertensão pulmonar grave, doença pulmonar restritiva grave, insuficiência renal avançada, insuficiência cardíaca congestiva avançada, gravidez anterior com préeclâmpsia grave, eclampsia ou síndrome HELLP (hemólise, enzimas hepáticas elevadas, plaquetopenia) e acidente vascular encefálico recente (DALAL DS, et al., 2019). As mulheres devem preferencialmente planejar a gravidez em um período de atividade mínima da doença. A contracepção apropriada deve ser usada para garantir este planejamento (TAVAKOLPOUR S e RAHIMZADEH G, 2016).

O LES é uma doença que pode afetar diversos órgãos em diferentes graus. Quando uma mulher que possui LES engravida, a doença pode trazer prejuízos à gestação, assim como a gestação pode trazer problemas ao curso da doença (DALAL DS, et al., 2019). O lúpus tem potencial de trazer complicações à gravidez causando CIUR, perda fetal, prematuridade e pré-eclâmpsia. Além disso, pode gerar também danos ao bebê, com o chamado lúpus neonatal, sendo sua manifestação mais grave o lúpus neonatal cardíaco (MAYNARD, et al., 2019).

A gestante com esta patologia deverá ser monitorada de perto por um médico, para controle adequado da doença e da saúde do feto, com a realização de exames clínicos e laboratoriais regulares, garantindo desta forma um bom desfecho para gestação (BĂLĂNESCU A, et al., 2017). A mulher com lúpus que deseja engravidar deve se programar e ser orientada por uma equipe multidisciplinar acerca da contracepção, dos riscos, do tratamento e do melhor momento para a gestação, minimizando os riscos e as chances de eventos desfavoráveis (BĂLĂNESCU A, et al., 2017).

\section{CONSIDERAÇÕES FINAIS}

O lúpus eritematoso sistêmico é uma doença que pode afetar indivíduos de ambos os sexos em qualquer fase da vida, mas costuma atingir preferencialmente pessoas do sexo feminino em idade fértil. Trata-se de uma doença autoimune, crônica e multissistêmica, capaz de afetar diversos órgãos e tecidos em diferentes graus. O lúpus pode trazer malefícios à mulher, a gestação e consequentemente ao bebê. A gravidez, por sua vez, pode exacerbar o lúpus, aumentando o número e a gravidade das crises da paciente. $O$ tratamento durante a gestação pode ser feito com alguns fármacos aprovados como hidroxicloroquina, azatioprina, ciclosporina e tacrolimus, sendo essas consideradas medicações seguras para uso durante todos os trimestres e também no período da lactação. Para reduzir a considerável morbimortalidade materna e fetal dessa doença em gestantes, recomenda-se à mulher um acompanhamento médico rigoroso pré e pós concepção e principalmente durante a gravidez.

\section{REFERÊNCIAS}

1. ANDREOLI L, et al. ULAR recommendations for women's health and the management of family planning, assisted reproduction, pregnancy and menopause in patients with systemic lupus erythematosus and/or antiphospholipid syndrome. Annals of the Rheumatic Disease, 2017; 76(3): 476-485.

2. BĂLĂNESCU A, et al. An ever-challenging relationship: lupus and pregnancy. Reumatologia, 2017; 55(1): 29-37.

3. BETZ RF, SPWCKER C. Pregnancy in systemic lupus erythematosus and antiphospholipid syndrome. Best Practice \& Research Clinical Rheumatology, 2017; 31(3): 397-414. 
4. DALAL DS, et al. Systemic Lupus Erythematosus and Pregnancy: A Brief Review. The Journal of Obstrectics and Gynecology of India, 2019; 69(2): 104-109.

5. HOCHBERG MC. Updating the american college of rheumatology revised criteria for the classification of systemic lupus erythematosus. Arthritis rheumatology 2017; 40(9): 1725.

6. HON KL, LEUNG AKC. Lúpus eritematoso neonatal. Autoimmune Diseases, 2012; 2012: 301274.

7. IMBERT GG, et al. Pregnancy and contraception in systemic and cutaneous lupus erythematosus. Annales de Dermatologie et de Venereologie, 2016; 143(10): 590-600.

8. JEON C, et al. Dermatologic conditions in patients of color who are pregnant. International Journal of Womens Dermatology, 2017; 3(1): 30-36.

9. KNIGHT CL, PIERCY CN. Management of systemic lupus erythematosus during pregnancy: challenges and solutions. Open Access Rheumatoogyl, 2017; 9: 37-53.

10. MARDER W. Update on pregnancy complications in systemic lupus erythematosus. Current Opinion, 2019; 31(6): 650-658.

11. MAYNARD S, et al. Pregnancy in Women with Systemic Lupus and Lupus Nephritis. Advences in Chronic Kidney Disease, 2019; 26(5): 330-337.

12. OSTENSE M, et al. Fourth International Conference on Sex Hormones, Pregnancy and Rheumatic Diseases consensus recommendations for anti- inflammatory, immunosuppressive and biological drugs and reproduction. Arthritis Research \& Therapy, 2016; 8(3): 209.

13. PASTORE DEA, et al. A Critical Review on Obstetric Follow-up of Women Affected by Systemic Lupus Erythematosus. Revista Brasileira de Ginecologia e Obstetrícia, 2018; 40(4): 209-224.

14. TAVAKOLPOUR S, RAHIMZADEH G. New Insights into the Management of Patients with Autoimmune Diseases or Inflammatory Disorders During Pregnancy. Scandinavian Journal of Immunology, 2016; 84(3): 146-9.

15. WU J, et al. Management and outcomes of pregnancy with or without lupus nephritis: a systematic review and metaanalysis. Therapeutics and Clinical Risk Management, 2018; 14: 885-901.

16. YOKOGAWA N, et al. Neonatal lupus erythematosus. Nihon Rinsho Meneki Gakkai Kaishi, 2017; 40(2): $124-130$. 